\title{
The Effectiveness of Rectal Aspiration Biopsy in the Diagnosis of Hirschsprung's Disease
}

\author{
Cemal Bilir ${ }^{1}$, mustafa onur oztan ${ }^{2}$, ayşe gülden diniz ${ }^{3}$, tunc ozdemir ${ }^{4}$, ali sayan ${ }^{4}$, and \\ gokhan koyluoglu ${ }^{2}$ \\ ${ }^{1}$ Bakircay Universitesi Tip Fakultesi \\ ${ }^{2}$ Izmir Katip Celebi University Faculty of Medicine \\ ${ }^{3}$ İzmir Democracy University \\ ${ }^{4}$ Republic of Turkey Ministry of Health Izmir Provincial Health Directorate Izmir \\ University of Health Sciences Tepecik Training and Research Hospital
}

February 20, 2021

\begin{abstract}
Purpose: In this prospective cross secitonal study, we aimed to investigate the adequacy, sensivity and specifity of rectal aspiration biopsy samples as the standart diagnosis of patients suspected HD for the histopathological diagnosis Material and methods: The study was performed between november 2016 and march 2018. We prospectively included 24 patients aged between 0-3 years that suspicious with HD to the study. After Rectosigmoid index (RSI) were calculated according to the contrast enhanced colon graphy, Patients who RSI $<1$ were performed rectal aspiration biopsy with the suspicious of HD. All patients' clinical features, treatment options, complications, laboratory results and radiological findings were recorded for further analyses. Biopsy specimens were evaluated by the specialist patholog and findings were recorded. Results: There were no ganglion on the specimens of rectal aspiration biopsy in 10(\% 41.6) patients and diagnosed as HD. Ganglion cell was detected in $5(\% 20.8)$ patients and diagnosis of HD was excluded. Inadequate or suspicious biopsy specimens for histopathological evaluation was observed in $9(\% 37.5)$ patients. If the biopsy volume was greater than $4 \mathrm{~mm} 3$, sensitivity was $80 \%$ and specificity was $66.67 \%$ in diagnosis of $\mathrm{HH}(\mathrm{AUC}=0.789)$; If the Submucosa / Mucosa ratio is greater than 0.75 or the submucosa is greater than 0.42 , the sensitivity and the specificity were $86.67 \%$ and $66.67 \%$, respectively. Conclusion: In this prospective cross-sectional study, we demonstrated that rectal aspiration biopsy in the diagnosis of HD is easily feasible, safe, has higher sensitivity and specificity, and lower complication rates.
\end{abstract}

\section{The Effectiveness of Rectal Aspiration Biopsy in the Diagnosis of Hirschsprung's Disease}

\section{ABSTRACT}

Purpose: In this prospective cross secitonal study, we aimed to investigate the adequacy, sensivity and specifity of rectal aspiration biopsy samples as the standart diagnosis of patients suspected HD for the histopathological diagnosis.

Material and methods: The study was performed between november 2016 and march 2018. We prospectively included 24 patients aged between 0-3 years that suspicious with HD to the study. After Rectosigmoid index (RSI) were calculated according to the contrast enhanced colon graphy, Patients who RSI $<1$ were performed rectal aspiration biopsy with the suspicious of HD. All patients' clinical features, treatment options, complications, laboratory results and radiological findings were recorded for further analyses. Biopsy specimens were evaluated by the specialist patholog and findings were recorded. 
Results: There were no ganglion on the specimens of rectal aspiration biopsy in 10(\%41.6) patients and diagnosed as HD. Ganglion cell was detected in 5(\%20.8) patients and diagnosis of HD was excluded. Inadequate or suspicious biopsy specimens for histopathological evaluation was observed in $9(\% 37.5)$ patients. If the biopsy volume was greater than $4 \mathrm{~mm} 3$, sensitivity was $80 \%$ and specificity was $66.67 \%$ in diagnosis of HH $($ AUC $=0.789)$; If the Submucosa / Mucosa ratio is greater than 0.75 or the submucosa is greater than 0.42 , the sensitivity and the specificity were $86.67 \%$ and $66.67 \%$, respectively.

Conclusion: In this prospective cross-sectional study, we demonstrated that rectal aspiration biopsy in the diagnosis of HD is easily feasible, safe, has higher sensitivity and specificity, and lower complication rates.

Keywords: Hirschsprung Disease, Ganglion, Rectal Aspiration Biopsy, Rectal Biopsy

\section{What is known?}

- Hirschsprung's disease is the migration deficiency of neural crest cells during the development of the bowels in the fetal period.

- Disgnosis of Hirschsprung's disease is made by demonstrating the complete absence of enteric ganglion cells in the submucosal and myenteric plexuses of the distal colon with rectal aspiration biopsy.

- Procedures such as anal dilatation or rectal irrigation used in the diagnosis and treatment of Hirschsprung's disease might contribute to the insufficient biopsy.

\section{What is new?}

- Suction Rectal Biopsy method in the diagnosis of Hirschsprung's disease provides a great advantage over full-thickness rectal biopsy

- Performing suction rectal aspiration biopsy after 48 hours from the anal dilatation or rectal irrigation is not contribute to the diagnosis of Hirschsprung's disease about insufficient biopsy

- Suction rectal aspiration biopsy is useful for the diagnosis of Hirschsprung's disease, especially in newborns.

\section{Introduction}

Hirschsprung's disease (HD) occurs in the newborn and childhood which presents with signs of intestinal obstruction and constipation that could have fatal complications if not diagnosed and treated on time (1). The main underlying pathology of the disease is the migration deficiency of neural crest cells during the development of the bowels in the fetal period. Functional obstruction occurs due to the inability to a relaxation of an aganglionic colon segment in the $\mathrm{HH}$. Because of the non-functional colon segment, dilatation in the proximal intestinal segment occurs $(2-5)$.

Hirschsprung's disease occurs in approximately 1 in 5,000 live births, and male to female ratio ranges from 3: 1 to 4: 1 (6-8). Most patients are diagnosed during the newborn period. Patients are admitted to the hospital with symptoms of distal intestinal obstruction such as bilious vomiting, abdominal distention and inability to defecate in the first 24-48 hours of life (9). Sometimes, in infants with short seg

"ment HD, the disease may progress with mild symptoms, so that diagnosis may delay until childhood period. Only up to $10 \%$ of patients are diagnosed after the age of three $(10,11)$.

The diagnosis is made by demonstrating the complete absence of enteric ganglion cells in the submucosal and myenteric plexuses of the distal colon in pathology preparations. Rectal biopsy could be performed by two methods: full-thickness rectal biopsy (FTRB) and suction rectal biopsy (SRB) (12). Biopsies must be taken $2 \mathrm{~cm}$ above the dentate line to prevent the mislead the diagnosis because below of dentate line is a physiological aganglionic region (13).

Previous studies reported that anal dilatation, rectal irrigation and barium enema used in the diagnosis and treatment of HD might contribute to the insufficient biopsy taken due to the inflammation and oedema in the anorectal mucosa, but these confining factors could not be excluded due to the retrospective design of the studies (14). 
The primary purpose of our study is to compare the effectiveness of SRB, which is performed 48 hours after these procedures, with other studies. The secondary purpose of this prospective study is to determine the sufficiency rate of SRB samples for histopathological diagnosis and to calculate the sensitivity and specificity of SRB after the histopathological examination.

\section{Materials and Methods}

This study was performed after the approval of Izmir Kâtip Çelebi University Faculty of Medicine Clinical Research Ethics Committee (Ethics committee date: 09.02.2017, Ethics committee decision number: 16) between 15 November 2016 and 15 March 2018 at Izmir Health Sciences University Tepecik Training and Research Hospital Pediatric Surgery Clinic. Data of 24 patients aged between 0-3 years who were hospitalized in our clinic for advanced examination and treatment with a pre-diagnosis of HD were analyzed prospectively.

Laboratory and abdominal X-Ray radiographs of the patients were evaluated. Then contrast-enhanced colon graphies and 24th hours control X-Ray graphies (retention graphy) were examined and recorded (Figure 1). Rectosigmoid index (RSI) was calculated for all patients. In the RSI evaluation, the presence of the transition zone and the presence of the barium in the intestines on the radiograph after 24 hours were evaluated and recorded. Rectosigmoid index was calculated by proportioning the diameter of the widest part of the rectum to the diameter of the sigmoid colon on barium enema graphy. If the $\mathrm{RSI}<1$, it was considered in favour of HD (Figure 2)(15).

After the complaints of these patients such as abdominal distention and vomiting were treated by nasogastric decompression; SRB was performed at least 48 hours after anal dilatation or rectal irrigation procedures. The biopsy was performed at the bedside to the patients younger than 1-year-old without any anaesthesia and in the operating room with sedation $(0.01 \mathrm{mg} / \mathrm{kg}$ IV midazolam) to the patients older than 1-year-old.

Suction rectal biopsies were performed with Rbi2 biopsy kit (Aus systems Pty Ltd, Allenby Gardens, Australia) (Figure 3). For histopathological diagnosis, we took two samples which are taken from $2 \mathrm{~cm}$ proximal of the anocutaneous line that one from the posterior wall and other from the lateral wall. Negative pressure during the procedure was adjusted to be $150 \mathrm{~mm} \mathrm{H}_{2} \mathrm{O}$ (It was created by vacuuming $5 \mathrm{cc}$ in a 10-cc injector)(14). Two fresh samples were quickly taken to the pathology laboratory, rightafter the samples were fixed in $10 \%$ formaldehyde solution, and paraffin blocks were prepared. Sections 4-5 microns thick from the paraffin blocks were stained with hematoxylin-eosin and examined under a light microscope at a magnification of 100 - 400 x (Figures 4). Ret-oncoprotein (Figure 4) and neuron-specific enolase (NSE) staining was performed by immunohistochemical (IHC) method on samples whose ganglion cells and nerve plexuses could not be selected in a routine examination. In addition, all sections were evaluated by measuring the mucosal (M) and submucosal (SM) areas in NIS Elements Ver 4.30 digital imaging program of Nikon Eclipse Ni microscope (Figure 4).

\section{Results}

This study includes 24 patients that $14(58 \%)$ were female, and $10(48 \%)$ were male. In terms of age, 15 $(62.5 \%)$ of 24 patients were newborns (0-28 days), and 9 (37.5\%) were children between 1 month and 36 months. Meconium output was not observed in $16(66.7 \%)$ patients at the first 24 hours. $10(62.5 \%)$ of these 16 patients were diagnosed with HD after further examinations and evaluations. RSI was calculated as $<1$ in $7(29.1 \%)$ patients and [?]1 in $9(37.5 \%)$ patients. Adequate radiography could not obtain or evaluated due to the presence of previous ileostomy or colostomy in 8 (33.3\%) of the patients. Seven (29.1\%) patients with RSI less than one were diagnosed HD after subsequent examinations.

During the study, none of the patients developed rectal bleeding, intestinal perforation, sepsis or similar complications after SRB at the diagnosis stage.

No ganglion cells were found in the samples of $10(41.6 \%)$ patients in the pathological evaluation and diagnosed as HD. Ganglion cells were observed in the pathological examination of the samples in $5(20.8 \%)$ of the patients, and HD was excluded. Biopsy samples of 9 (37.5\%) patients were evaluated and reported as insufficient or suspicious samples for the pathological examination. 
FTRB was obtained from 4 (16.6\%) patients who were evaluated as inadequate and suspicious SRB, and HD diagnosis was excluded in these patients due to the presence of ganglion cells in the pathological examination of the samples. The other $5(20.8 \%)$ patients with insufficient results were discharged after their complaints regressed, and due to the symptoms were regressed by follow-up, we excluded the diagnosis of HD. Afterwards, patients were taken periodic follow-up for six months by outpatient clinic controls. During the follow-up period, no findings suggestive of HD were detected, and their families were informed and excluded from the follow-up. In addition, all patients were followed up with outpatient clinic controls after discharge for at least six months. From the SRB results obtained from 24 patients, the volume of the biopsy, the submucosa $(\mathrm{SM}) /$ mucosa $(\mathrm{M})$ ratio and the percentage of SM tissue in the preparation (SM\%) were calculated. The results were summarized in table 1 .

When we compared the preoperative SRB materials and postoperative resection materials, 10 (41.6\%) patients with preoperative ganglion negative SRB results showed aganglionosis in postoperative resection materials. In the pathological evaluation of samples in $5(20.8 \%)$ patients, the presence of a ganglion cell in SRB was reported. According to these results of 5 patients; due to the disappearance of symptoms in favour of HD in clinical observation, after 6 months of outpatient clinic follow-up, their families were informed, and they were excluded from the follow-up.

When Receiver Operating Characteristic (ROC) analysis was performed to compare SRB samples with pathology results and clinical examination results, it was calculated that if the biopsy volume was greater than $4 \mathrm{~mm}^{3}$, it had $80 \%$ sensitivity and $66.67 \%$ specificity in the diagnosis of HD (AUC $=0.789$ ); if the $\mathrm{SM} / \mathrm{M}$ ratio is greater than 0.75 or the $\mathrm{SM}$ is greater than 0.42 , it has been found that it has $86.67 \%$ sensitivity and $66.67 \%$ specificity ( $\mathrm{AUC}=0.748, \mathrm{AUC}=0.752$, respectively) (Table 2 ).

When the results of SRB were evaluated, samples of $15(62.5 \%)$ patients were sufficient, and $9(37.5 \%)$ patients were insufficient or suspicious. The mean volume of adequate biopsies was $8.4 \mathrm{~mm} 3, \mathrm{SM} / \mathrm{M}$ ratio 1.22, SM\% 0.52, respectively. The mean volume of inadequate biopsies was $4.7 \mathrm{~mm} 3, \mathrm{SM} / \mathrm{M}$ ratio $0.77, \mathrm{SM} \%$ 0.40, respectively. In terms of SM\% of patients, the difference between groups was statistically significant (p: 0.033) (Table 3).

When we evaluate the adequacy of the SRBs in newborn and children between one month and three years old; We calculated the SRB adequacy rate as $73.3 \%$ in newborns and $44.4 \%$ in children aged between one month and three years (Table 4).

\section{Discussion}

In this study, we aimed to measure the adequacy of the SRB method applied to take the rectal biopsy sample, which is the gold standard diagnostic method for HD diagnosis, and in addition to report the results of the test. We found that the SRB results obtained for HD were sufficient for diagnosis. $62.5 \%$ of samples receiving SRB were sufficient, and $37.5 \%$ of samples were insufficient or suspicious. Insufficient sampling rates have been reported at different rates in several studies in the literature. Although debates on this issue continue, studies reported insufficient sampling rates at the range between $11-50 \%$ (16-22). The main difference between these studies is the design of the biopsy procedure that has taken with different procedures. When we investigate the studies in the literature, we observed that most of the studies have low insufficient material rate, 3 or 4 samples were taken in each biopsy procedure, and also biopsy was repeated if the submucosal tissue was small. Mostly, studies reported that 1-4 samples must be taken for each biopsy procedure. In many studies, at least three samples were taken (17-22). Although we took two samples from each patient in our study, we consider that our insufficient sampling rate was acceptable. However, we considered that the reason why this rate was higher when compared to other studies in the literature was that we only obtained two samples in each procedure, did not have SRB for the second time, and that we have just started to apply this protocol in our clinic. In addition, the biopsy of our two patients who were reported suspicious but considered insufficient may have increased this rate.

In our study, when samples containing sufficient tissue and insufficient samples were examined to confirm or exclude the disease in the pathological examinations of biopsies; the average volume of sufficient samples was 
$8.4 \mathrm{~mm}^{3}$ and $4.7 \mathrm{~mm}^{3}$ for insufficient samples. Although these values are not statistically significant, it is noteworthy that the volume of sufficient samples is high. There are limited studies in the literature regarding the volume measurement of samples. In the study of Muise, the average volume obtained from SRB samples was reported as $14.8+-7.8 \mathrm{~mm} 3(14)$. Several studies reported that an adequate biopsy sample should be at least $3.5 \mathrm{~mm}$ in diameter $(17,23,24)$. The increase in the volume of the samples contributes to make a decision of diagnosis, but the depth of the biopsy also is another important criterion for the diagnosis. In the histopathological examinations of the samples in our study, the average percentage of submucosal tissue of sufficient samples was found to be $52 \%$, and the average of the insufficient samples was $40 \%$. The difference was statistically significant $(\mathrm{p}=0.033)$. Although there are few prospective studies in this direction in the literature, it is remarkable that retrospective studies reported that the rate of submucosa in the biopsy tissue should not be less than $35-50 \%$ among the inclusion criteria for the samples $(14,16,17)$. In our study, similar rates were reported with these studies in the literature in terms of both the volume of SRB samples and the percentage of submucosal tissue.

In our study, we found that $73.3 \%$ of the biopsy tissues obtained from the newborn age group were sufficient for histopathological examination, while $44.4 \%$ of the patients aged between one month and three years were considered sufficient. Although this difference is not statistically significant, tissue adequacy in SRB was found to be higher in newborns than in older babies in our study. When the studies in the literature are examined, different results draw attention when the diagnostic feature of SRB according to age groups is evaluated. While some studies reported that there was no difference in the diagnostic yield of SRB between age groups, several studies reported that insufficiency rate was higher in infants younger than 1.5 months compared to infants older than 1.5 months $(5,22)$. In another study, it was reported that the tissue adequacy rate was higher in babies older than one year compared to babies older than one year old (25). In some studies, it has been reported that the tissue adequacy ratio of SRB decreases after the age of $3(20,26,27)$. Since different results have been reported in the literature on this issue, there is no consensus yet. Because in older children, obtaining biopsy under sedation and the presence of hypertrophic nerve fibres in the pathological evaluation of the tissue obtained is a positive factor in diagnosis, previous enterocolitis attacks and thicker intestinal mucosa could be considered as a negative factor and may play a role in obtaining different results in each study. Although the immaturity of the ganglion cells in the neonatal period and the lack of hypertrophic nerve fibres are factors that challenge the pathologist, an experienced pathologist can make a definitive diagnosis with a sample that has reached a sufficient amount of submucosal tissue. It is well-known that full-thickness rectal biopsy procedure is more difficult, especially in the newborn period compared to older ages. In our study, a higher material adequacy rate in newborn period could be achieved with the SRB method, and lower complication rates were observed in all-aged patients. These results show that the SRB technique is an easily applicable and safe method in newborn periods.

In patients with a pre-diagnosis of HD, often anal dilatation and rectal irrigation are recommended methods in terms of both diagnosis and treatment during the decision of biopsy procedure (4). We planned a prospective study by eliminating this suspicious procedure for the first time in the literature, with the suspicion that both procedures may have an oedema-forming effect on the rectum wall and may prevent sufficient submucosal tissue obtain during the SRB procedure. We concluded that our insufficient material rates were parallel to similar studies in the literature. As a result, we revealed that giving patients a 48-hour period without anal intervention before SRB does not provide any benefit, and it is possible to perform biopsy whenever desired. When 9 of the biopsies obtained from 24 patients included in the study, which were considered as insufficient material, were not included in the evaluation, the specificity and sensitivity of SRB from 15 patients was found to be $100 \%$. It has been reported that the sensitivity of SRB in the diagnosis of HD is $88-93 \%$ and its specificity is $95-99 \%(22,23,28-30)$. In the study of Nicole, no false negative or false positive results were reported, similar to our study $(28)$.

The limitations of our study were; when compared to previous studies, the reason why our study was $100 \%$ accurate, and there was no margin of error caused by the low number of cases. However, we consider that excluding suspicious SRB results from the evaluation and working with an experienced pathologist for the diagnosis of HD increased the accuracy of the study results. Further prospective-randomized double-blind 
studies including large-patient population, it may be expected that the sensitivity and specificity rate, which is $100 \%$, will decrease slightly. Therefore, this high rate supports the opinions that the SRB technique should be preferred over FTRB.

\section{Conclusion}

This study showed that the SRB method in the diagnosis of HD provides a great advantage over fullthickness rectal biopsy because it could be performed safely and without anaesthesia due to the very low rate of serious complications in both newborn period and older children. In addition, when insufficient material was obtained in HD diagnosis neglected, considering that the sensitivity and specificity rates were very high, could be applied quickly and easily for the diagnosis of HD. And also, it provides an advantage when performing definitive operations after possible HD, rectal biopsy, which is the gold standard method for the diagnosis of $\mathrm{HD}$, is primarily we consider that it should be obtained with the rectal aspiration biopsy method.

\section{Acknowledgement}

This article was prepared from the thesis of Dr. Cemal Bilir. Dr. Gokhan Koyluoğlu consulted this thesis.

\section{References}

1. Martucciello G, Prato AP, Puri P, Holschneider AM, Meier-Ruge W, Jasonni V, et al. Controversies concerning diagnostic guidelines for anomalies of the enteric nervous system: a report from the fourth International Symposium on Hirschsprung's disease and related neurocristopathies. Journal of pediatric surgery. 2005;40(10):1527-31.

2. Parisi MA. Hirschsprung Disease Overview-RETIRED CHAPTER, FOR HISTORICAL REFERENCE ONLY. GeneReviewsß[Internet]: University of Washington, Seattle; 2015.

3. Badner JA, Sieber W, Garver K, Chakravarti A. A genetic study of Hirschsprung disease. American journal of human genetics. 1990;46(3):568.

4. Dasgupta R, Langer JC. Hirschsprung disease. Current problems in surgery. 2004;41(12):942-88.

5. Daniel H. Hirschsprung's disease and related neuromuscular disorders of the intestine. Pediatric surgery. 1998:1381-424.

6. Suita S, Taguchi T, Ieiri S, Nakatsuji T. Hirschsprung's disease in Japan: analysis of 3852 patients based on a nationwide survey in 30 years. Journal of pediatric surgery. 2005;40(1):197-202.

7. Best KE, Addor MC, Arriola L, Balku E, Barisic I, Bianchi F, et al. Hirschsprung's disease prevalence in Europe: a register based study. Birth Defects Research Part A: Clinical and Molecular Teratology. 2014;100(9):695-702.

8. Ieiri S, Suita S, Nakatsuji T, Akiyoshi J, Taguchi T. Total colonic aganglionosis with or without small bowel involvement: a 30-year retrospective nationwide survey in Japan. Journal of pediatric surgery. 2008;43(12):2226-30.

9. Khan A, Vujanic G, Huddart S. The constipated child: how likely is Hirschsprung's disease? Pediatric surgery international. 2003;19(6):439-42.

10. of the North CGC. Evaluation and treatment of constipation in infants and children: recommendations of the North American Society for Pediatric Gastroenterology, Hepatology and Nutrition. Journal of Pediatric Gastroenterology and Nutrition. 2006;43(3):e1-e13.

11. Arshad A, Powell C, Tighe M. Hirschsprung's disease. Bmj. 2012;345.

12. Friedmacher F, Puri P. Current practice patterns of rectal suction biopsy in the diagnostic work-up of Hirschsprung's disease: results from an international survey. Pediatric surgery international. 2016;32(8):71722 . 
13. Martin LW, Torres AM. Hirschsprung's disease. Surgical Clinics of North America. 1985;65(5):1171-80.

14. Muise ED, Hardee S, Morotti RA, Cowles RA. A comparison of suction and full-thickness rectal biopsy in children. Journal of Surgical Research. 2016;201(1):149-55.

15. Garcia R, Arcement C, Hormaza L, Haymon M, Ward K, Velasco C, et al. Use of the recto-sigmoid index to diagnose Hirschsprung's disease. Clinical pediatrics. 2007;46(1):59-63.

16. Brady A-C, Saito JM, Lukas K, Guthrie T, Utterson EC, White FV, et al. Suction rectal biopsy yields adequate tissue in children. Journal of pediatric surgery. 2016;51(6):966-9.

17. Croffie JM, Davis MM, Faught PR, Corkins MR, Gupta SK, Pfefferkorn MD, et al. At what age is a suction rectal biopsy less likely to provide adequate tissue for identification of ganglion cells? Journal of pediatric gastroenterology and nutrition. 2007;44(2):198-202.

18. Meinds RJ, Kuiper G-A, Parry K, Timmer A, Groen H, Heineman E, et al. Infant's age influences the accuracy of rectal suction biopsies for diagnosing of Hirschsprung's disease. Clinical Gastroenterology and Hepatology. 2015;13(10):1801-7.

19. de Arruda Lourenção PL, Takegawa BK, Ortolan EV, Terra SA, Rodrigues MA. Does calretinin immunohistochemistry reduce inconclusive diagnosis in rectal biopsies for Hirschsprung disease? Journal of pediatric gastroenterology and nutrition. 2014;58(5):603-7.

20. Hayes CE, Kawatu D, Mangray S, LeLeiko NS. Rectal suction biopsy to exclude the diagnosis of Hirschsprung disease. Journal of pediatric gastroenterology and nutrition. 2012;55(3):268-71.

21. Yang WI, Oh J-T. Calretinin and microtubule-associated protein-2 (MAP-2) immunohistochemistry in the diagnosis of Hirschsprung's disease. Journal of pediatric surgery. 2013;48(10):2112-7.

22. de Haro Jorge I, Bellver PP, Masip VJ, García LS, Farres TR, Pallejà DC, et al. effectiveness of calretinin and role of age in the diagnosis of Hirschsprung disease. Pediatric surgery international. 2016;32(8):723-7.

23. Noblett HR. A rectal suction biopsy tube for use in the diagnosis of Hirschsprung's disease. Journal of pediatric surgery. 1969;4(4):406-9.

24. Qualman SJ, Jaffe R, Bove KE, Monforte-Muñoz H. Diagnosis of Hirschsprung disease using the rectal biopsy: multi-institutional survey. Pediatric and Developmental Pathology. 1999;2(6):588-96.

25. Lall A, Gupta D, Bajpai M. Neonatal Hirschsprung's disease. The Indian Journal of Pediatrics. 2000;67(8):583-8.

26. Alizai N, Batcup G, Dixon M, Stringer M. Rectal biopsy for Hirschsprung's disease: what is the optimum method? Pediatric surgery international. 1998;13(2-3):121-4.

27. Campbell P, Noblett HR. Experience with rectal suction biopsy in the diagnosis of Hirschsprung's disease. Journal of pediatric surgery. 1969;4(4):410-5.

28. Sharp NE, Pettiford-Cunningham J, Shah SR, Thomas P, Juang D, Peter SDS, et al. The prevalence of Hirschsprung disease in premature infants after suction rectal biopsy. journal of surgical research. 2013;184(1):374-7.

29. De Lorijn F, Reitsma JB, Voskuijl WP, Aronson DC, Fiebo J, Smets AM, et al. Diagnosis of Hirschsprung's disease: a prospective, comparative accuracy study of common tests. The Journal of pediatrics. $2005 ; 146(6): 787-92$.

30. Kobayashi H, Li Z, Yamataka A, Lane GJ, Miyano T. Rectal biopsy: what is the optimal procedure? Pediatric surgery international. 2002;18(8):753-6.

\section{TABLES}

Table 1. Histopathological evaluation results of biopsy specimens 


\begin{tabular}{llll}
\hline & Volume $\left(\mathrm{mm}^{3}\right)$ & $\mathrm{SM} / \mathrm{M}$ & $\mathrm{SM} \%$ \\
\hline Number & 24 & 24 & 24 \\
Average & 7.04 & 1.05 & 0.47 \\
Standard deviation & 4.63 & 0.55 & 0.12 \\
(SD) & & & \\
Minimum & 3.00 & 0.36 & 0.26 \\
Maximum & 24.00 & 2.41 & 0.70 \\
\hline
\end{tabular}

Table 2. ROC analysis results for histopathological evaluations (+ PV: Positive predictive value, - PV: Negative predictive value, AUC: Area under the curve, CI: Confidence interval)

\begin{tabular}{|c|c|c|c|c|c|c|c|c|}
\hline & $\begin{array}{l}\text { Cut-off } \\
\text { value }\end{array}$ & $\begin{array}{l}\text { Cut-off } \\
\text { value }\end{array}$ & $\begin{array}{l}\text { Sensitivity } \\
(95 \% \text { GA })\end{array}$ & $\begin{array}{l}\text { Specificity } \\
(95 \% \mathrm{GA})\end{array}$ & $+\mathrm{PV}$ & \multicolumn{2}{|c|}{$-\mathrm{PV}$} & AUC \\
\hline SM\% & $>0.42$ & $>0.42$ & $\begin{array}{l}86.67(59.5- \\
98.0)\end{array}$ & $\begin{array}{l}66.67 \\
(30.1- \\
92.1)\end{array}$ & 81.3 & \multicolumn{2}{|c|}{75.0} & 0.752 \\
\hline $\mathrm{SM} / \mathrm{M}$ & $>0.75$ & $>0.75$ & $\begin{array}{l}86.67 \\
(59.5- \\
98.0)\end{array}$ & $\begin{array}{l}66.67 \\
(30.1- \\
92.1)\end{array}$ & 81.3 & \multicolumn{2}{|c|}{75.0} & 0.748 \\
\hline Volume & Volume & $>4$ & $\begin{array}{l}80.00 \\
(51.9- \\
95.4)\end{array}$ & $\begin{array}{l}66.67 \\
(30.1- \\
92.1)\end{array}$ & 80.0 & \multicolumn{2}{|c|}{66.7} & 0.789 \\
\hline & \multicolumn{2}{|r|}{ Biopsy adequacy } & Mean & & \multicolumn{2}{|c|}{ Standard deviation } & \multicolumn{2}{|l|}{$\mathrm{P}$-value } \\
\hline \multicolumn{2}{|l|}{ Volume $\left(\mathrm{mm}^{3}\right)$} & $\begin{array}{l}\text { Insufficient }(\mathrm{n}=9) \\
\text { Sufficient }(\mathrm{n}=15)\end{array}$ & $\begin{array}{l}4.77 \\
8.40\end{array}$ & & $\begin{array}{l}1.56 \\
5.35\end{array}$ & \multicolumn{3}{|c|}{0.62} \\
\hline $\mathrm{SM} / \mathrm{M}$ & & $\begin{array}{l}\text { Insufficient }(\mathrm{n}=9) \\
\text { Sufficient }(\mathrm{n}=15)\end{array}$ & $\begin{array}{l}0.77 \\
1.22\end{array}$ & & $\begin{array}{l}0.46 \\
0.54\end{array}$ & \multicolumn{3}{|c|}{0.55} \\
\hline $\mathrm{SM} \%$ & & $\begin{array}{l}\text { Insufficient }(n=9) \\
\text { Sufficient }(n=15)\end{array}$ & $\begin{array}{l}0.40 \\
0.52\end{array}$ & & $\begin{array}{l}0.11 \\
0.11\end{array}$ & \multicolumn{2}{|r|}{0.33} & \\
\hline
\end{tabular}

Table 3. Histopathological adequacy assessment of biopsy

Table 4. Biopsy adequacy values according to age groups

\begin{tabular}{|c|c|c|c|c|c|c|}
\hline & & & Age groups & Age groups & & \\
\hline \multirow{3}{*}{ Biopsy adequacy } & & & Newborn & 1 month-3 years & Total & P-value \\
\hline & Insufficient & number $(\%)$ & $4(26.7)$ & $5(55.6)$ & $9(37.5)$ & 0.212 \\
\hline & Sufficient & number $(\%)$ & $11(73.3)$ & $4(44.4)$ & $15(62.5)$ & \\
\hline Total & Total & number $(\%)$ & $15(100)$ & $9(100)$ & $24(100)$ & \\
\hline
\end{tabular}

\section{FIGURE LEGENDS}

Figure 1 - X-ray imaging of the patient with suspected Hirschsprung disease ( A: Broad-based air-fluid 
levels in the abdominal radiography of a patient with abdominal distension, B: Anterior/posterior barium enema graphy, C: Lateral barium enema graphy, D: Control X-ray radiography after 24 hours from barium enema application)

Figure 2 - A . Normal RSI measurement. The ratio of the widest diameter (RR') of the rectum to the widest diameter (SS') of the sigmoid colon is greater than or equal to 1. B. Anormal RSI: Hirschsprung's disease: Ratio is less than 1

Figure 3- Rbi2 suction rectal biopsy system

Figure 4- A. Ganglion cells in nerve plexuses $(\mathrm{HE} \times 100)$,B. Ganglion cell $(\mathrm{HE} \times 400)$, C. ganglion cell stained with ret-oncoprotein $(\mathrm{DAB} \times 400)$, D. measurement of mucosa/submucosa areas

\section{Hosted file}

Figure 1.pdf available at https://authorea.com/users/396907/articles/509975-theeffectiveness-of-rectal-aspiration-biopsy-in-the-diagnosis-of-hirschsprung-s-disease

\section{Hosted file}

figure 2.pdf available at https://authorea.com/users/396907/articles/509975-theeffectiveness-of-rectal-aspiration-biopsy-in-the-diagnosis-of-hirschsprung-s-disease

\section{Hosted file}

Figure 3.pdf available at https://authorea.com/users/396907/articles/509975-theeffectiveness-of-rectal-aspiration-biopsy-in-the-diagnosis-of-hirschsprung-s-disease

Hosted file

Figure 4.pdf available at https://authorea.com/users/396907/articles/509975-theeffectiveness-of-rectal-aspiration-biopsy-in-the-diagnosis-of-hirschsprung-s-disease 